\title{
One for all and all for one
}

\author{
Our aim at Communications Earth \& Environment is to disseminate and debate the science of our planet \\ and our Solar System. Together with the community of scientists, we can create an open resource of \\ data, analyses and insights that will deepen our understanding of the Earth and beyond.
}

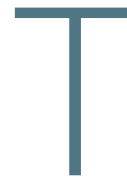

he Earth and environmental sciences are central to guiding humanity's pathway into the future. Over the past decades, it has become ever more important to transition from exploitation to stewardship of the Earth. Climate change, pollution and the use of resources such as oil, gas, metals and minerals, must be put on a sustainable footing if severe degradation of our environment is to be avoided. At the same time, we want to push for a deeper understanding of how our planet as well as other bodies in the Solar System have evolved in response to external and internal disturbances. At Communications Earth \& Environment, we invite all Earth, environmental and planetary scientists to contribute their knowledge and findings. It is our ambition to offer scrutiny and discussion within a thriving community of researchers across the many and diverse fields of geoscience that we cover. Where appropriate, we will also cover their societal context.

Take the lock-downs in the face of the COVID-19 pandemic. The global restrictions on mobility and activity have dampened, but not resolved, many impacts of human action on the environment, bringing sharply into focus how far we have moved away from a sustainable society. For example, although the lock-downs in a third of China's cities substantially improved air quality, concentration of fine particulate matter still exceeded levels considered safe by the World Health Organization-by more than a factor of four ${ }^{1}$. Similarly, the impact of the global restrictions on human activities on 2020 carbon emissions has been estimated ${ }^{2}$ to fall between as little as 4.2 and $7.5 \%$. Even during the peak of the confinements, the temporary decrease in daily fossil $\mathrm{CO}_{2}$ emissions took the world back by no more than $17 \%$, equivalent to the levels of about 2006-and still a long way off international climate targets.
Scientific investigations with such direct implications for our lives need openness and accountability. In the climate sciences, just over 10 years ago a surge of freedomof-information requests for the data behind climate research marked a turning point. Although the requests were part of a politically motivated campaign that culminated in the hacking and illegal release of thousands of private emails, in the wake of the 'Climategate' affair the community of climate scientists recognised that allowing scrutiny of data and methods is a necessary condition for public trust in science. Other fields in the Earth, environmental and planetary sciences have followed suit.

Because the research we publish matters, at Communications Earth \& Environment, we require data deposition in a publically accessible repository for our articles. We work with our authors to make data FAIR: Findable, Accessible, Interoperable, and Reusable. We offer unlimited space in the Methods section, and ask authors to explain them to a level that allows others to reproduce exactly what they did. And last, not least, our articles are Open Access.

We want our community of researchers to come from all regions on Earth and all walks of life. We commit to publishing high-quality research and commissioning overview articles and opinion pieces from a diverse set of authors. Since we opened for submissions in February, we have received submission from 37 countries, and we are pleased to report that the demographics of our authors match those of our reviewers in terms of geography; the US, China, and Germany and the UK as joint third are our leading contributors, both in terms of authorship and in terms of reviewers. We are actively encouraging gender balance in our reviewers: $31 \%$ of our reviewers gave their gender as female, compared to $65 \%$ as male (the remainder choose not to disclose); these numbers compare favourably 


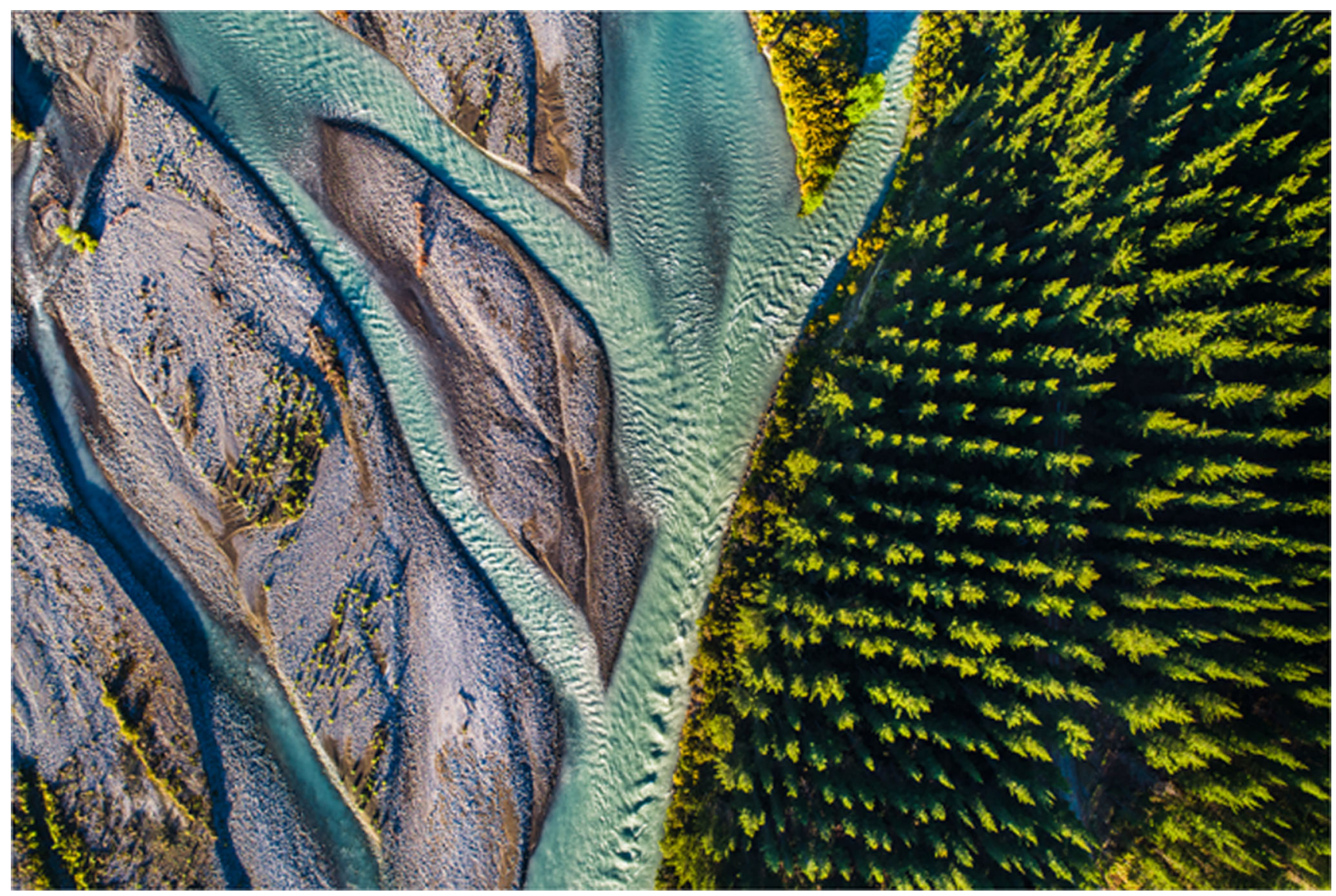

Image credit: nazar_ab

with data collected at Nature Geoscience, for example, where $25 \%$ of reviewers were female in a survey ${ }^{3}$ over 5 months in 2019.

We would like to improve further on diversity. We emphatically invite those from underrepresented groups to join us on the journey ahead, as an author, or by expressing an interest to work with us as a reviewer or as an Editorial Board Member. In addition, we would like to promote progress towards diversity and inclusion in the geosciences, and encourage anyone with relevant unique and compelling ideas and results to contribute to the journal. Indeed, we are pleased to include in our first batch of articles a primary research study ${ }^{4}$ that evaluates interventions aimed at retaining undergraduate women in geoscience-related careers.

Our launch articles cover a broad range of exciting geoscience topics, from high to low latitudes, from atmosphere to solid Earth, and from the past into the future, with articles investigating Greenland ice dynamics; turbidity currents offshore from river deltas; abrupt climate change during the last glacial period; the characteristics of volatile organic compounds over Amazonia; and the healing process in fault rocks following earthquakes. We look forward to filling any gaps in time and space as we add article by article in the coming weeks, months and years.

We are exploring, and will continue to explore, ways to counteract existing biases in science and society. We encourage all our readers to give their views in our survey on universal-as opposed to optional-Double Blind Peer Review. And we have adjusted our editorial processes in response to the COVID-19 pandemic: we ask authors who have been particularly hard hit to alert us in their cover letter, so that we can expedite handling their paper. We are also relaxing our assessment of novelty where two articles from independent groups have been developed in parallel.

It has been an exciting journey for the entire editorial team-in-house and Editorial Board Members alike-to get to the point where we can proudly present our first batch of articles, and we would like to thank all the authors and reviewers who have contributed already. The journal launch is only the beginning. We are immensely looking forward to working closely with the community of geoscience researchers in years to come. Together we can make a difference.

Published online: 13 August 2020

\section{References}

1. He, G., Pan, Y. \& Tanaka, T. The short-term impacts of COVID-19 lockdown on urban air pollution in China. Nat. Sustain. https://doi.org/ 10.1038/s41893-020-0581-y (2020).

2. Le Quere, C. et al. Temporary reduction in daily global $\mathrm{CO}_{2}$ emissions during the COVID-19 forced confinement. Nat. Clim. Change 10, 647-653 (2020)

3. Anonymous. Quality and equality in review. Nat. Geosci. 12, 681 (2019).

4. Hernandez, P. et al. Inspiration, inoculation, and introductions are all critical to successful mentorship for undergraduate women pursuing geoscience careers. Commun. Earth Environ. https://doi.org/10.1038/s43247-020-0005-y (2020).

(c) (i) Open Access This article is licensed under a Creative Commons Attribution 4.0 International License, which permits use, sharing, adaptation, distribution and reproduction in any medium or format, as long as you give appropriate credit to the original author(s) and the source, provide a link to the Creative Commons license, and indicate if changes were made. The images or other third party material in this article are included in the article's Creative Commons license, unless indicated otherwise in a credit line to the material. If material is not included in the article's Creative Commons license and your intended use is not permitted by statutory regulation or exceeds the permitted use, you will need to obtain permission directly from the copyright holder. To view a copy of this license, visit http:// creativecommons.org/licenses/by/4.0/.

() Springer Nature Limited 2020 